\title{
DISCOVERY OF PULSED X-RAY EMISSION FROM THE SMALL MAGELLANIC CLOUD TRANSIENT RX J0117.6-7330
}

\author{
D. J. Macomb,${ }^{1,2}$ M. H. Finger,${ }^{2,3}$ B. A. Harmon,${ }^{3}$ R. C. Lamb,${ }^{4}$ and T. A. Prince ${ }^{4}$ \\ Received 1999 January 13; accepted 1999 April 14; published 1999 May 25
}

\begin{abstract}
We report on the detection of pulsed, broadband, X-ray emission from the transient source RX J0117.6-7330. The pulse period of $22 \mathrm{~s}$ is detected by the ROSAT/PSPC instrument in a 1992 September 30-October 2 observation and by the Compton Gamma-Ray Observatory/BATSE instrument during the same epoch. Hard X-ray pulsations are detectable by BATSE for approximately 100 days surrounding the ROSAT observation (1992 August 28December 8). The total directly measured X-ray luminosity during the ROSAT observation is $1.0 \times 10^{38}(d / 60$ $\mathrm{kpc}^{2} \mathrm{ergs} \mathrm{s}^{-1}$. The pulse frequency increases rapidly during the outburst, with a peak spin-up rate of $1.2 \times$ $10^{-10} \mathrm{~Hz} \mathrm{~s}^{-1}$ and a total frequency change of $1.8 \%$. The pulsed percentage is $11.3 \%$ from $0.1-2.5 \mathrm{keV}$, increasing to at least 78\% in the 20-70 keV band. These results establish RX J0117.6-7330 as a transient Be binary system.
\end{abstract}

Subject headings: accretion, accretion disks — stars: individual (RX J0117.6-7330) - X-rays: general

\section{INTRODUCTION}

The PSPC instrument on board the ROSAT spacecraft made an 8.98 ks observation of the Small Magellanic Cloud on 1992 September 30-October 2, leading to the discovery (Clark, Remillard, \& Woo 1996) of RX J0117.6-7330, a bright Xray source within $5^{\prime}$ of SMC X-1. The source was not detected in an observation of the SMC $1 \mathrm{yr}$ earlier and was found 246 days later to be dimmer by more than 2 orders of magnitude. The derived X-ray luminosity was $2.3 \times 10^{37}(d / 60$ $\mathrm{kpc})^{2} \operatorname{ergs~s}^{-1}(0.2-2.5 \mathrm{keV}$; Clark, Remillard, \& Woo 1997). Spectral analysis showed the source to be relatively soft, with a power-law index of around 2.7 (although a power law is not the best-fit model). A Fourier analysis did not reveal any significant periodicities, with the authors lamenting an increase in spectral noise at frequencies below $0.1 \mathrm{~Hz}$.

The companion star first suggested by Clark et al. (1996) was observed by Charles, Southwell, \& O’Donoghue (1996) optically in 1996 January. They determined that the B1-2 star of magnitude 14.2 proposed as the companion showed a strong IR excess and Balmer lines and a reddening typical of an $\mathrm{OB}$ star in the SMC, thus strengthening the association of the Xray source with the SMC. They also argue that the luminosity and companion type indicates that the $\mathrm{X}$-ray source is a neutron star (Coe et al. 1998). However, Clark et al. (1997) hypothesize that the system could harbor a black hole based on the $e$-folding X-ray decay time of 44 days, the rather soft spectrum, and the lack of any neutron star rotation period in the X-ray analysis.

We have performed a reanalysis of the ROSAT/PSPC data and have coupled it with hard X-ray observations by the Compton Gamma-Ray Observatory (CGRO)/BATSE instrument. In $\S 2$, we present evidence for X-ray emission pulsed at a $22.067 \mathrm{~s}$ period that definitively establishes the X-ray source as a neutron star. We show the frequency history for RX J0117.6-7330 during this 100 day outburst, which reveals an extremely large average frequency derivative of $8.9 \times$ $10^{-11} \mathrm{~Hz} \mathrm{~s}^{-1}$ corresponding to a spin-up timescale of $16 \mathrm{yr}$.

\footnotetext{
${ }^{1}$ Laboratory for High-Energy Astrophysics, NASA/Goddard Space Flight Center, Greenbelt, MD 20771.

${ }^{2}$ Astrophysics Program, University Space Research Association.

${ }^{3}$ Space Sciences Laboratory, ES 84, NASA/Marshall Space Flight Center, Huntsville, AL 35812.

${ }^{4}$ Space Radiation Laboratory, California Institute of Technology, Pasadena, CA 91125.
}

The frequency derivative peaked at $1.2 \times 10^{-10} \mathrm{~Hz} \mathrm{~s}^{-1}$, with the pulse frequency increasing by $1.8 \%$ during the BATSE observations. The broadband X-ray pulse shapes and pulsed flux are calculated in $\S 3$. Section 4 summarizes our findings and discusses RX J0117.6-7330 in the context of the Be class of high-mass X-ray binaries.

\section{PERIODICITY SEARCH}

RX J0117.6-7330 was 5' from the center of the PSPC field of view during an $8985 \mathrm{~s}$ exposure taken from MJD 48,895.7 to MJD 48,897.6 (MJD = JD -2,400,000.5). We determine a total source count rate of $4.43 \pm 0.03$ counts $\mathrm{s}^{-1}$ from 0.1 to $2.4 \mathrm{keV}$. Photons in a circle of radius $1^{\prime}$ surrounding the source position R.A., decl. $(\mathrm{J} 2000)=\left(01^{\mathrm{h}} 17^{\mathrm{m}} 36^{\mathrm{s}},-73^{\circ} 30^{\prime} 00^{\prime \prime}\right)$ were extracted and barycentered using standard FTOOLS software. A total of 33,989 photons were available for timing analysis.

These photons were collected into $5 \mathrm{~ms}$ bins over the full length of the observation, a time span of $162 \mathrm{ks}$. A fast Fourier transform (FFT) of the resultant time series was then calculated, sensitive to periods in the range from $10 \mathrm{~ms}$ to $81,000 \mathrm{~s}$ with the power per channel normalized to unity using the average power for all frequencies above $0.01 \mathrm{~Hz}$. No frequency derivatives were included at this point of the analysis. A peak of power 30, normalized as above, at a frequency of 0.090825(2) $\mathrm{Hz}$ was evident in this analysis and warranted further study despite increased noise due to the complicated ROSAT exposure-induced window function and spacecraft wobble.

Verification of the pulsed signal comes from an archival search of data from the BATSE instrument on CGRO. BATSE is capable of nearly continuous monitoring of hard X-ray sources using both the Earth occultation technique (Harmon et al. 1993) and timing techniques (Bildsten et al. 1997). A search of archival BATSE FFT results identified a possibly related outburst some 60 days after the 1992 ROSAT observation at a frequency of $0.0914825(2) \mathrm{Hz}$, with a frequency derivative of $9.7(2) \times 10^{-11} \mathrm{~Hz} \mathrm{~s}^{-1}$ consistent with the direction of the SMC. With follow-up epoch-folding-based searches of the BATSE data during the ROSAT observation, it became apparent that the originally detected frequency was the second harmonic of the pulse frequency. From 6 days of BATSE data centered on the ROSAT observation, a barycentric pulse frequency of $0.045316682(55) \mathrm{Hz}$ (MJD 48,896.65) and frequency derivative of 9.81(8) $\times 10^{-11} \mathrm{~Hz} \mathrm{~s}^{-1}$ were determined. Folding the 


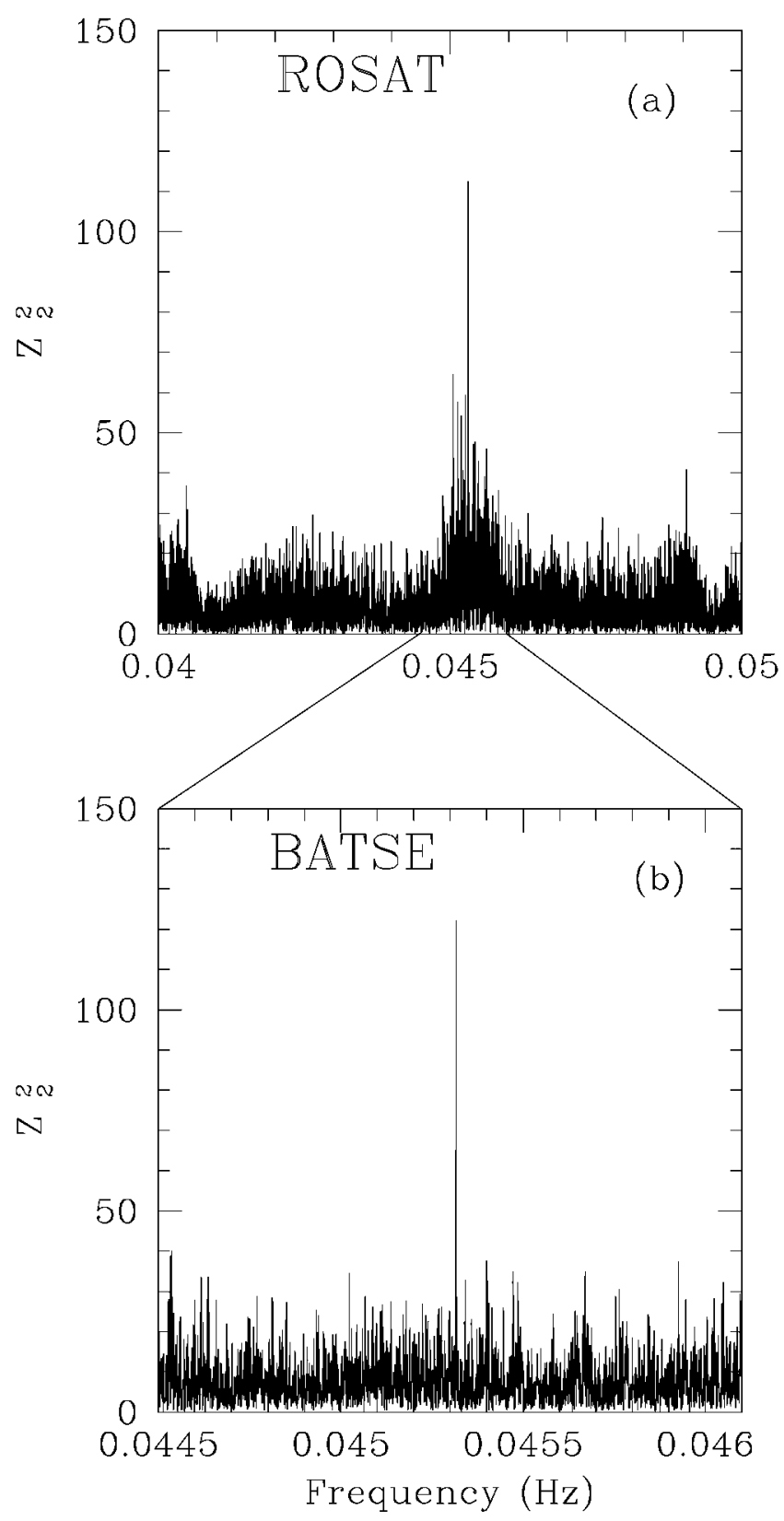

FIG. 1.-ROSAT power distribution encompassing the first harmonic of the pulsed frequency. The BATSE distribution is for a narrow range around the ROSAT detection frequency.

ROSAT data with this frequency derivative gives a peak power at $0.0453168(3) \mathrm{Hz}$, consistent with the BATSE pulse period. Figure 1 shows the resultant ROSAT power spectrum calculated using the $Z_{2}^{2}$ statistic (Buccheri et al. 1983) over a narrow frequency range utilizing the above-stated frequency derivative. Also plotted is the same statistic for the BATSE data at frequencies near the ROSAT signal.

The results of searching the BATSE DISCLA channel 1 data (20-50 keV, $1.024 \mathrm{~s}$ resolution) from 1992 August 16 (MJD 48,850) to 1993 January 12 for pulsations from RX J0117-7730 are presented in Figure 2. These searches were performed in 6 day intervals, using an epochfolding-based search (see Bildsten et al. 1997) that used only the first and second harmonic of the pulse profile and incorporated a search in both pulse frequency and frequency deriv-

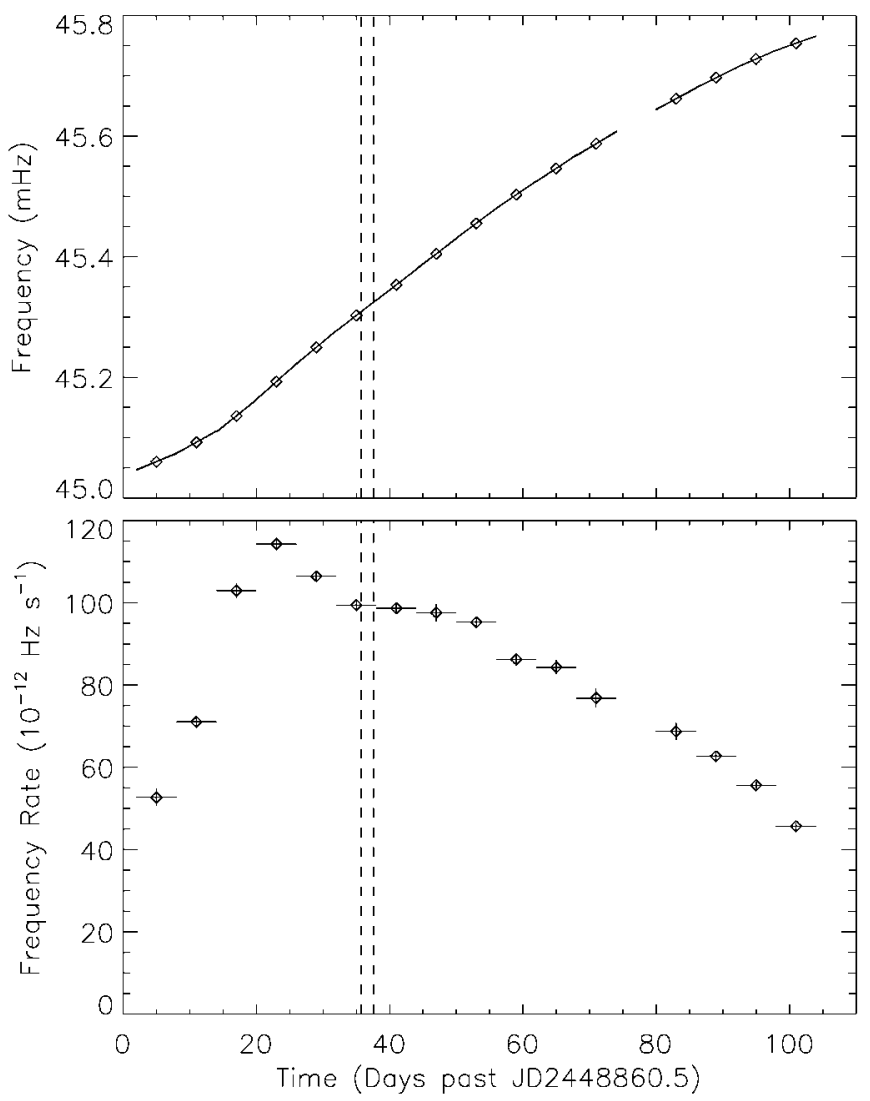

FIG. 2.-BATSE pulse timing analysis of RX J0117.6-7330. The top panel is the frequency history, and the bottom panel is the frequency rate history. Both plots use the best-fit values for 6 day intervals, with the ROSAT observation date shown by the dashed line.

ative. For intervals in which pulsations were detected, the pulse frequency and frequency derivative are shown. The frequency derivative peaks at $1.2 \times 10^{-10} \mathrm{~Hz} \mathrm{~s}^{-1} 25$ days before the 1992 ROSAT observations. The pulse frequency increases by $1.8 \%$ during the outburst. The signal is present for approximately 100 days, starting about 34 days before the ROSAT/PSPC observation.

\section{BROADBAND PULSE PROFILE AND FLUX}

Using the measured frequency and frequency derivative, we can construct the pulse profile for RX J0117.6-7330 for both soft and hard X-ray energies. Figure 3 shows both the ROSAT/ PSPC pulse profile for 1992 September 30-October 2 (MJD 48,895-48,897) and the CGRO/BATSE profile for MJD 48,893.65-48,899.65. Both data sets use the pulse phase model based on the BATSE data. The optimal ROSAT frequency and frequency derivatives are slightly different. While the very high frequency derivative coupled with the long integration times ( 6 days for BATSE, 2 days for ROSAT) make absolute timing comparisons slightly problematic, there is no evidence for a loss of coherence in the BATSE folding, and the pulse phases from the two instruments should be directly comparable. Figure 3 shows the pulse shapes for both energy ranges using an epoch at phase zero of MJD 48,896.65. An extra phase offset of 0.14 was added in order to make the BATSE minimum correspond to phase zero. The overall profile shapes are similar. The peaks and minima of the light curves are generally in phase, but the primary peak in the ROSAT 

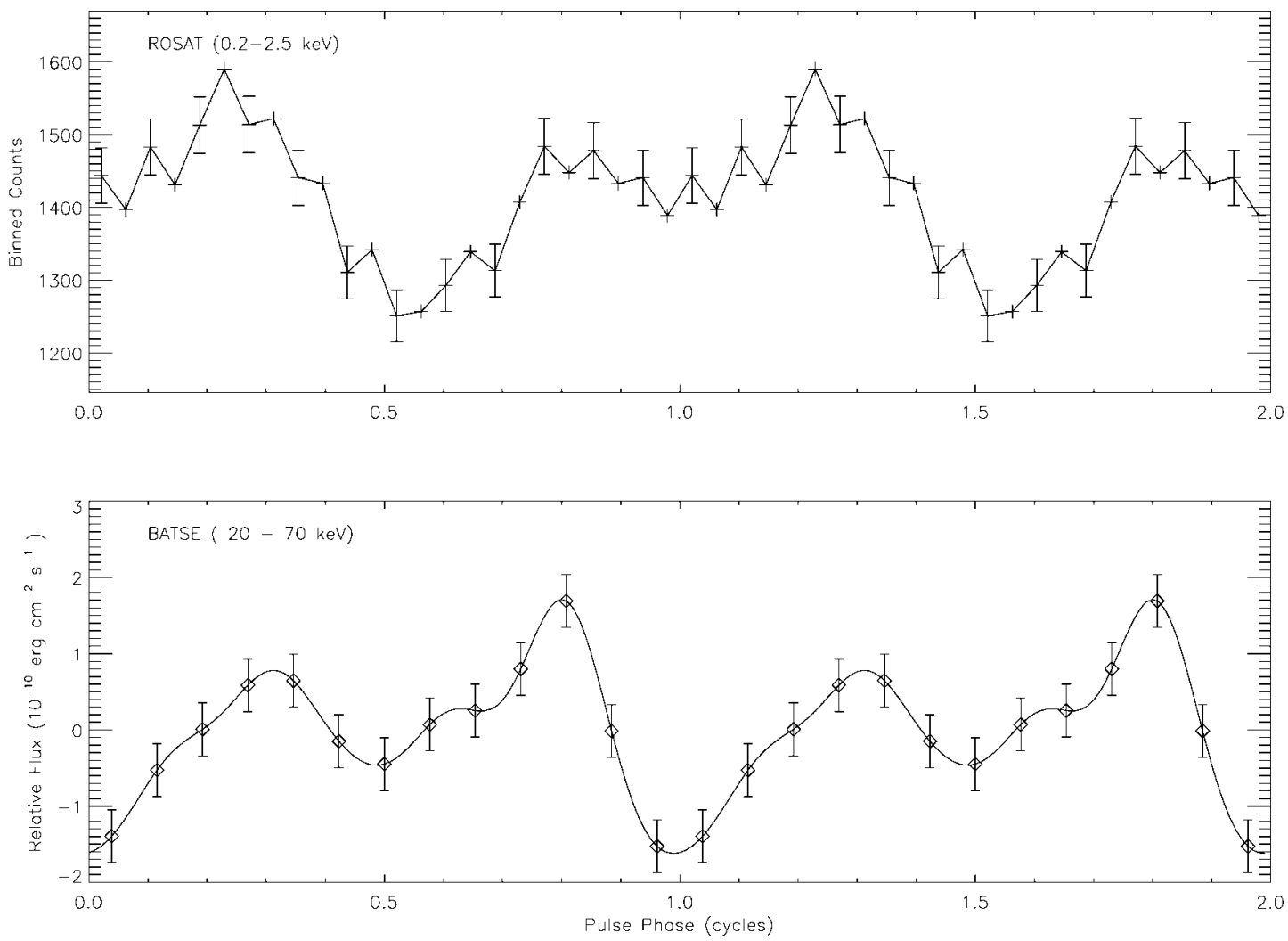

FIG. 3.-Folded light curves for ROSAT 0.1-2.4 keV (top) and BATSE 20-70 keV (bottom) data. The BATSE profile is limited to six Fourier harmonics, resulting in the smooth shape. Flux errors are given for a set of approximately independent phases.

energy range becomes the secondary peak in the BATSE range. Similarly, primary and secondary minima are interchanged.

We have tested our timing analysis methods using contemporaneous ROSAT/PSPC and CGRO/BATSE observations of PSR 1509-58. In this case, we find pulse profiles with shapes and radio-phase offsets consistent with previously published results (Greiveldinger et al. 1995; Ulmer et al. 1993). The overall pulse shape for RX J0117.6-7330 in both energy ranges is similar, so it is not out of the question that the shape as a whole has simply shifted. Apparent phase shifts of simple profiles in different energy bands have been previously observed. For example, the $1.2-2.3$ and $18.4-27.5 \mathrm{keV}$ profiles of GS 0834-430 observed with Ginga by Akoi et al. (1992) show a complex evolution with energy. It would be somewhat coincidental, however, for the phase shift to be such that the minima and maxima still coincide. At this point, we consider the peaks to be aligned, with the relative strengths to be changing.

From these pulse profiles, one may determine the pulsed flux and pulsed fraction. Using XSPEC, we calculate a total flux of $(5.1 \pm 0.3) \times 10^{-11} \mathrm{ergs} \mathrm{cm}^{-2} \mathrm{~s}^{-1}$. Similar to Clark et al. (1997), we find that the best fit to the ROSAT spectrum is a combination of power law and bremsstrahlung or blackbody, although a straight power-law fit of index $2.65 \pm 0.07$ is not much worse. Of the 33,989 total counts extracted for the light curve, 3829 comprise the pulsed excess, giving a pulsed percentage of $11.3 \% \pm 2.3 \%$. This corresponds to a total pulsed flux in the $0.2-2.5 \mathrm{keV}$ band of $(5.6 \pm 1.7) \times 10^{-12} \mathrm{ergs} \mathrm{cm}^{2}$ $\mathrm{s}^{-1}$. In the BATSE energy range, the phase-averaged pulsed fraction is more difficult to assess. An occultation analysis of RX J0117.6-7330 detects a clear signal over the same time frame as the epoch-folding analysis. However, source confusion could significantly contribute to the detected flux. For a 20 day period around the time of the ROSAT observation, the average total flux is $0.012 \pm 0.02 \mathrm{~cm}^{-2} \mathrm{~s}^{-1}$. This corresponds to an energy flux of $(2.3 \pm 0.4) \times 10^{-10} \mathrm{ergs} \mathrm{cm}^{-2} \mathrm{~s}^{-1}$ (assuming a power-law index 3.0, 20-100 keV). The BATSE pulsed spectrum is best fit by a thermal bremsstrahlung model with temperature $18 \pm 3 \mathrm{keV}$. The integrated $20-70 \mathrm{keV}$ pulsed flux is $(1.8 \pm 0.2) \times 10^{-10} \mathrm{ergs} \mathrm{cm}^{-2} \mathrm{~s}^{-1}\left[7.8 \times 10^{37}(D / 60 \mathrm{kpc})^{2}\right.$ ergs $\mathrm{s}^{-1}$ ]. These values provide us with a lower limit to the pulsed fraction in the $20-70 \mathrm{keV}$ range of $78 \%$ (a lower limit, since the measured occultation flux is considered to be an upper limit to total emission because of possible source confusion and the occultation analysis went up to $100 \mathrm{keV}$ ). The directly measured flux in the $0.2-2.5$ and $20-70 \mathrm{keV}$ bands alone is then at least $(2.3 \pm 0.2) \times 10^{-10} \mathrm{ergs} \mathrm{cm}^{2} \mathrm{~s}^{-1}[(1.0 \pm$ $\left.0.1) \times 10^{38}(D / 60 \mathrm{kpc})^{2} \mathrm{ergs} \mathrm{s}^{-1}\right]$.

\section{DISCUSSION}

X-ray pulsations at a period of $22.07 \mathrm{~s}$ from the bright Xray transient RX J0117.6-7330 have been detected in both the 0.1-2.4 and 20-70 keV energy bands. This confirms the identity of the X-ray source as a neutron star rather than a black hole. Transient X-ray pulsars are typically found in Be systems, which is consistent with, and supports the identification of, the proposed optical counterpart.

The CGRO/BATSE detection allows long-term monitoring of the outburst from this source. The hard X-rays are detectable for over 100 days starting 34 days before the ROSAT observation began. A large average spin-up is present over the du- 
ration of the outburst, resulting in a $1.8 \%$ change in frequency. The peak frequency changes appear 10-15 days before the ROSAT observation and approximately 20 days after the outburst began. Some of the frequency derivative may be caused by the binary orbit. Some of the variations seen in Figure 2 near the peak of the frequency derivative may be an orbital signature. Such variations are weak, however, compared to the overall accretion-induced changes in frequency.

The high intrinsic spin-up rates imply that an accretion disk is present about the neutron star, as is generally seen in the "giant" or type I outbursts of Be/X-ray pulsars (Bildsten et al. 1997). The measured luminosity is at least $1.0 \times 10^{38} \mathrm{ergs} \mathrm{s}^{-1}$ during the ROSAT observation in the combined 0.2-2.5 and $20-70 \mathrm{keV}$ bands. The peak frequency derivative was about $15 \%$ higher than during the ROSAT observation. However, we have no data in the energy range from $2.5-20 \mathrm{keV}$. With a complicated ROSAT X-ray spectrum and a changing pulse fraction, it is difficult to extrapolate our results to this energy range. However, it is likely that the luminosity in this range is comparable to that measured in the $0.2-2.5$ and $20-70 \mathrm{keV}$ ranges. Thus, the peak luminosity, when adjusted for higher frequency derivative and $2-20 \mathrm{keV}$ emission, is $\geq 1 \times 10^{38} \mathrm{ergs} \mathrm{s}^{-1}$ and was probably higher than the conventional Eddington limit. This is consistent with the trend for Magellanic Cloud binaries to be much brighter on average than their Galactic counterparts-probably due to the absence of metals, which supply accretion-inhibiting absorption (Clark et al. 1978; van Paradijs $\&$ McClintock 1995). From the peak spin-up rate and standard accretion theory (Bildsten et al. 1997 and references therein), we can obtain a lower limit on the peak luminosity of $\sim 2.5 \times 10^{38} \mathrm{ergs} \mathrm{s}^{-1}$. This is consistent with the highest luminosities of X-ray binaries in the SMC (van Paradijs \& McClintock 1995). It is inconsistent with the source being in our Galaxy.

The pulse profiles in the two energy bands are similar in that they both have a double-peaked structure. However, the main and secondary peaks are interchanged. X-ray binaries typically have pulse profiles that are often strongly energy dependent (White, Swank, \& Holt 1983). In this case, the doublepeaked light curve in both energy bands shows the same peakto-peak separation of 0.5 . The pulsed fraction increases from $11 \%$ in soft X-rays to at least $78 \%$ in hard X-rays. If the overall morphology of the pulses is indeed the same, with the exception of the relative strengths of the two peaks, this may indicate a high magnetic field since at energies above the cyclotron energy, the pulse shape is expected to change significantly (e.g., see Sturner \& Dermer 1994).

This project made use of software and data provided by the High-Energy Astrophysics Archival Research Center (HEASARC) located at Goddard Space Flight Center. This work was supported at Caltech in part by NASA NAG5-3239.

\section{REFERENCES}

Aoki, T., et al. 1992, PASJ, 44, 641

Bildsten, L., et al. 1997, ApJS, 113, 367

Buccheri, R., et al. 1983, A\&A, 128, 245

Charles, P. A., Southwell, K. A., \& O’Donoghue, D. 1996, IAU Circ. 6305

Clark, G., Doxsey, R., Lie, F., Jernigan, J. G., \& van Paradijs, J. 1978, ApJ, 221, L37

Clark, G., Remillard, R., \& Woo, J. 1996, IAU Circ. 6282 . 1997, ApJ, 474, L111

Coe, M. J., Buckley, D. A. H., Charles, P. A., Southwell, K. A., \& Stevens, J. B. 1998, MNRAS, 293, 43

Greiveldinger, C., Caucino, S., Massaglia, S., Ogelman, H., \& Trussoni, E. 1995, ApJ, 454, 855
Harmon, A., et al. 1993, in AIP Conf. Proc. 280, Compton Gamma-Ray Observatory, ed. M. W. Friedlander, N. Gehrels, \& D. J. Macomb (New York: AIP), 314

Sturner, S. J., \& Dermer, C. D. 1994, A\&A, 284, 161

Ulmer, M. P., et al. 1993, ApJ, 417, 738

van Paradijs, J., \& McClintock, J. E. 1995, in X-Ray Binaries, ed. W. H. G. Lewin, J. van Paradijs, \& E. P. J. van den Heuvel (Cambridge: Cambridge Univ. Press), 113

White, N. E., Swank, J. H., \& Holt, S. S. 1983, ApJ, 270, 711 